\title{
ASO Visual Abstract: Early Diagnosis of Anastomotic Leakage After Gastric Cancer Surgery via Analysis of Inflammatory Factors in Abdominal Drainage
}

Jinyao Shi, PhD, Zhouqiao Wu, MD, PhD, Xiaolong Wu, MD, Fei Shan, MD, Yan Zhang, MSE, Xiangji Ying, MPH, MS, Ziyu Li, MD, and Jiafu Ji, MD, PhD

Gastrointestinal Cancer Center, Key Laboratory of Carcinogenesis and Translational Research (Ministry of Education), Peking University Cancer Hospital and Institute, Hai-Dian District, Beijing, People's Republic of China

Abnormally upregulated intra-abdominal inflammatory factors were significantly associated with anastomotic leakage after gastric surgery. A new assessment method was constructed based on the concentration of inflammatory factors within the abdominal drainage, which showed positive performance and was prospectively validated (https://doi.org/10.1245/s10434-021-10763-y).

\section{Early Diagnosis of Anastomotic Leakage After Gastric Cancer Surgery Via Analysis of Inflammatory Factors in Abdominal Drainage}

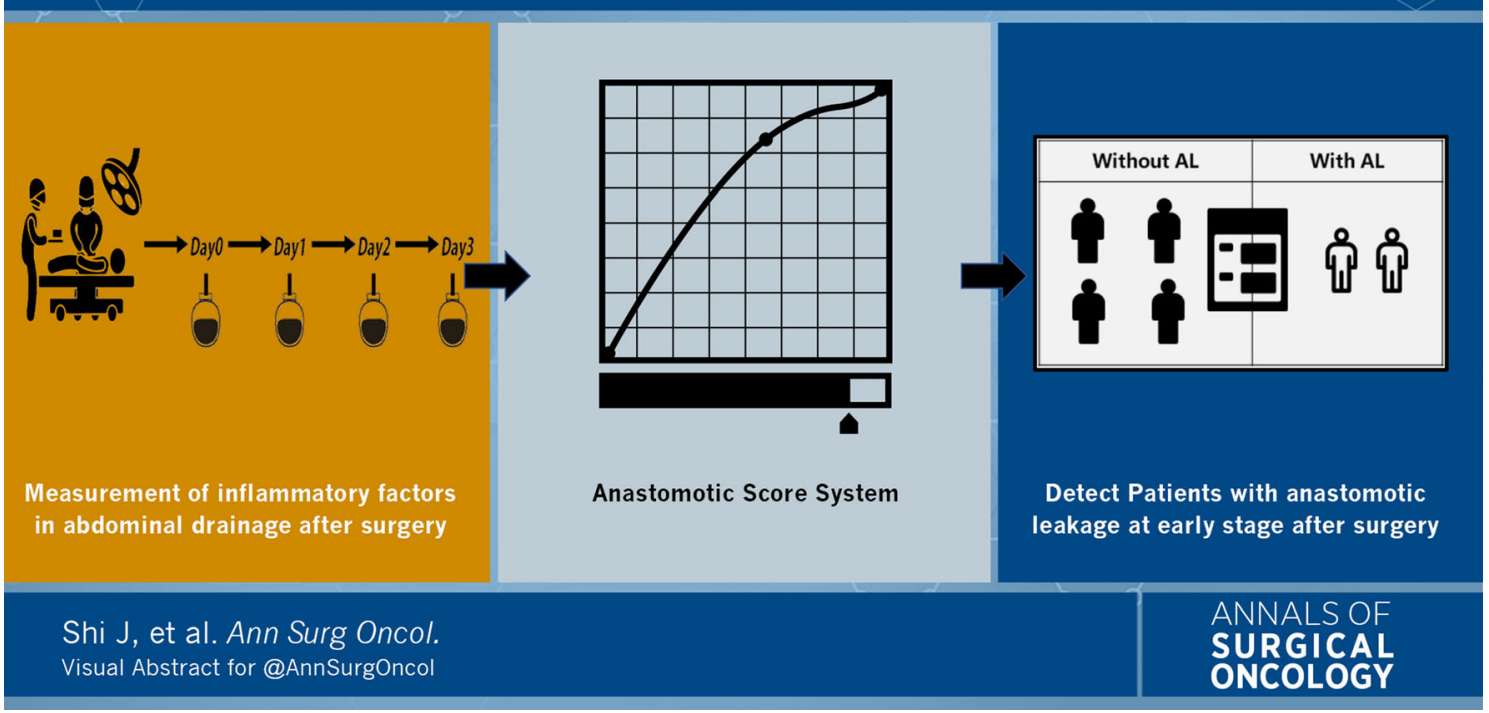

Jinyao Shi and Zhouqiao Wu have contributed equally to this work.

(C) Society of Surgical Oncology 2021

Published Online: 6 October 2021

Z. Li, MD

e-mail: ziyu_li@hsc.pku.edu.cn

J. Ji, MD, PhD

e-mail: jijiafu@hsc.pku.edu.cn

\section{DISCLOSURE}

The authors declare that there is no conflict of interest.

Publisher's Note Springer Nature remains neutral with regard to jurisdictional claims in published maps and institutional affiliations. 